\title{
Die Umweltverbandsklage nach dem Regierungsentwurf zur Anpassung des UmwRG an europa- und völkerrechtliche Vorgaben
}

Fabian Michl

Die gesetzliche Regelung der Umweltverbandsklage sieht sich nicht zuletzt seit den Empfehlungen des Aarhus Convention Compliance Committee (ACCC) vom 20.12.2013 bestätigt durch die 5. Vertragsstaatenkonferenz am 2.7.2014 - und dem Urteil des EuGH im Vertragsverletzungsverfahren gegen Deutschland vom 15.10 .2015 erheblichem Anpassungsdruck aus dem Völker- und Europarecht ausgesetzt. Am 22.6.2016 hat die Bundesregierung einen Gesetzentwurf beschlossen, mit dem die völker- und europarechtlichen Vorgaben eins zu eins in das deutsche Recht umgesetzt werden sollen. Der Beitrag erläutert und bewertet die wesentlichen Änderungen, die der Gesetzentwurf im Recht der Umweltverbandsklage vorsieht.

\section{Völker- und europarechtlicher Anpassungsdruck}

Die Regelungen des Umweltrechtsbehelfsgesetzes (UmwRG) über die Umweltverbandsklage implementieren die völkerrechtlichen Vorgaben der Aarhus Konvention (AK) sowie die einschlägigen EU-Richtlinien (UVP- und IE-RL) ${ }^{1}$, die wiederum die Anforderungen der AK in das Unionsrecht übernehmen.

\subsection{Art. 9 Abs. 2 und 3 AK}

Art. 9 AK stellt in seinen Absätzen 2 und 3 abgestufte Anforderungen an den Gerichtszugang der betroffenen Öffentlichkeit. Nach Art. 2 Nr. 5 AK sind Teil der betroffenen Öffentlichkeit insbesondere die Umweltverbände.

Art. 9 Abs. 2 verlangt nach einer Möglichkeit für Umweltverbände, die materiellrechtliche und verfahrensrechtliche Rechtmäßigkeit von Maßnahmen gerichtlich überprüfen zu lassen, für welche nach Art. 6 AK i. V. m. Anh. I zur AK eine Öffentlichkeitsbeteiligung vorgesehen ist. Die Vertragsparteien können diese nach dem Konventionstext umfassende Rechtmäßigkeitskontrolle auf andere Maßnahmen erweitern, die von sonstigen Bestimmungen der Konvention (z. B. Art. 7 AK: Öffentlichkeitsbeteiligung bei umweltbezogenen Plänen, Programmen und Politiken) erfasst sind. In der derzeitigen Fassung des UmwRG ist eine solche Erstreckung nicht vorgesehen; § 1 Abs. 1 Satz 1 Nr. 1 und 2 UmwRG betrifft nur Entscheidungen, die

Fabian Michl, LL.M. (Edin.), Rechtsanwalt in Regensburg und wissenschaftlicher Mitarbeiter am Lehrstuhl für Öffentliches Recht, insb. deutsches und europäisches Verwaltungsrecht, Prof. Dr. Gerrit Manssen, Universität Regensburg.

${ }^{1}$ Art. 11 RL 2011/92/EU = UVP-RL; Art. 25 RL 2010/75/EU = IE-RL. 
unter Art. 6 AK fallen, und deckt damit zugleich den Regelungsbereich der UVPund IE-RL ab.

Nach Art. 9 Abs. 3 AK ist - jenseits des Geltungsbereichs von Art. 6 AK - eine Anfechtungsmöglichkeit gegen behördliche und private Maßnahmen vorzusehen, die gegen umweltbezogene Bestimmungen des innerstaatlichen Rechts verstoßen. Die Rechtmäßigkeitskontrolle ist insoweit also auf umweltbezogene Rechtsvorschriften beschränkt. Art. 9 Abs. 3 AK hat in der bisherigen Fassung des UmwRG keinen Niederschlag gefunden. Einzig auf Entscheidungen nach dem Umweltschadensgesetz wurde der Anwendungsbereich des UmwRG ausgedehnt (§ 1 Abs. 1 Satz 1 Nr. 3 UmwRG), allerdings nicht unter dem Eindruck des Art. 9 Abs. 3 AK. ${ }^{2}$ Im Gegenteil ging der Gesetzgeber bei der Ratifizierung der AK davon aus, dass Art. 9 Abs. 3 AK bereits vollständig durch bestehendes Recht umgesetzt sei. ${ }^{3}$ Die übrigen gesetzlichen Rechtsschutzmöglichkeiten der Umweltverbände beschränken sich derzeit auf die naturschutzrechtliche Verbandsklage nach § 64 BNatSchG und die Verletztenklage nach den allgemeinen Vorschriften ( $\S \S 42$ Abs. 2 Hs. 2, 113 Abs. 1 Satz 1 VwGO).

\subsection{Umsetzungsdefizite}

Von Anfang an stand das UmwRG wegen der nur halbherzigen Umsetzung der völker- und europarechtlichen Vorgaben in der Kritik, die sich - heute ${ }^{4}$ - im Wesentlichen auf die folgenden Problemkreise bezieht. ${ }^{5}$

\subsubsection{Anwendungsbereich (§ 1 Abs. 1 UmwRG)}

Nach den „Feststellungen und Empfehlungen“ des ACCC vom 20.12.2013 hat Deutschland den Vorgaben des Art. 9 Abs. 3 AK nicht hinreichend Rechnung getragen, da in vielen Bereichen des umweltbezogenen nationalen Rechts nicht sichergestellt sei, dass Umweltverbände eine Klage erheben könnten, ohne eine Verletzung in eigenen Rechten geltend machen zu müssen. ${ }^{6}$ Zwar seien die Vertragsparteien nicht gehalten, in ihrem nationalen Recht eine Popularklage

\footnotetext{
${ }^{2}$ Ursprünglich war die entsprechende Anwendung des UmwRG in § 11 Abs. 2 USchG angeordnet; zur Übernahme in das UmwRG vgl. BT-Drs. 17/10957, S. 15.

${ }^{3}$ BT-Drs. 16/2497, S. 46.

${ }^{4}$ Hauptkritikpunkt an der Umweltverbandsklage bis 2013 war die sog. Schutznormakzessorietät der Klagebefugnis und der Begründetheitsprüfung; vgl. dazu Berkemann, DVBl. 2016, 205.

${ }^{5}$ Vgl. zu den „Baustellen“ des UmwRG Berkemann, DVBl. 2016, 205 ff.

${ }^{6}$ ACCC/C/2008/31 (Germany), Tz. 91 ff., abrufbar unter http://www.unece.org/fileadmin/DAM/env/pp/compliance/C2008-

31/Findings/C31Germany_findings_advancedunedited.pdf; zuletzt abgerufen am 18.7.2016.
} 
vorzusehen, mit der jeder umweltbezogene Rechtsverstoß von jedermann geltend gemacht werden könne. Die Voraussetzungen, unter denen Rechtsschutz gewährt würde, dürfe aber auch nicht so eng sein, dass sie praktisch alle Mitglieder der betroffenen Öffentlichkeit, einschließlich der Umweltverbände, daran hinderten, Maßnahmen anzugreifen, die gegen nationale umweltbezogene Rechtsvorschriften verstießen. ${ }^{7}$ Die strikte Anwendung der Schutznormtheorie im Rahmen der Verletztenklage stelle eine solche praktische Rechtsschutzverhinderung dar, da zahlreiche umweltbezogene Rechtsverstöße keine subjektiven Rechte berührten. ${ }^{8} \mathrm{Da}$ die naturschutzrechtliche Verbandsklage nicht alle umweltbezogenen Bestimmungen erfasst, kam das ACCC zu dem Ergebnis, dass in Deutschland - trotz vereinzelter Lockerungen der Schutznormtheorie in der Rechtsprechung - für die Umweltverbände entgegen Art. 9 Abs. 3 AK keine hinreichenden Rechtsschutzmöglichkeiten bestünden. ${ }^{9}$ Die 5. Vertragsstaatenkonferenz pflichtete dieser Einschätzung bei und empfahl Deutschland, zusätzlich zur naturschutzrechtlichen Verbandsklage Rechtsschutzmöglichkeiten zu eröffnen, die den Anforderungen von Art. 9 Abs. 3 AK entsprächen. ${ }^{10}$

Der Anpassungsdruck sub specie Art. 9 Abs. 3 AK wird erhöht durch zwei Judikate des EuGH und des BVerwG, die noch vor den ACCC-Empfehlungen ergangen sind. ${ }^{11}$ Der EuGH hatte im sog. Slowakischen-Braunbären-Fall ${ }^{12}$ zum Artenschutzrecht entschieden, dass der nationale Richter, wenn eine unionsrechtlich geschützte Art betroffen sei, sein nationales Recht im Hinblick auf die Gewährung eines effektiven gerichtlichen Rechtsschutzes in den vom Umweltrecht der Union erfassten Bereichen so auszulegen habe, dass es so weit wie möglich im Einklang mit den in Art. 9 Abs. 3 AK festgelegten Zielen stehe. ${ }^{13}$ Auch wenn die genaue Bedeutung des Urteils unklar geblieben ist, lässt sich daraus doch eine Tendenz des EuGH zur Ausweitung der Umweltverbandsklage ablesen. ${ }^{14}$ Dieser Tendenz ist das BVerwG in seinem Urteil vom 5.9.2013 gefolgt, in dem es aus $\S 47$ Abs. 1 BImSchG

\footnotetext{
${ }^{7}$ ACCC/C/2008/31 (Germany), Tz. 92.

${ }^{8}$ ACCC/C/2008/31 (Germany), Tz. 95.

${ }_{9}^{9}$ ACCC/C/2008/31 (Germany), Tz. 96 ff.

${ }_{10} \mathrm{~V} / 9 \mathrm{~h}$ ECE/MP.PP/2014/CRP.4, S. 1 f., abrufbar unter

https://www.unece.org/fileadmin/DAM/env/pp/mop5/Documents/Insession_docs/ece.mp.pp.2014.crp.4.pdf; zuletzt abgerufen am 18.7.2016.

${ }^{11}$ Dazu Greim, BayVBl. 2014, 517, 518 ff.

${ }^{12}$ EuGH, Urt. v. 8.3.2011 - C-240/09, Slg. 2011, I-01255, NuR 2011, 346.

${ }^{13}$ EuGH, Urt. v. 8.3.2011 - C-240/09, Slg. 2011, I-01255, Rdnr. 50, NuR 2011, 346, 350.

${ }^{14}$ Vgl. die krit. Analyse von Greim, Rechtsschutz bei Verfahrensfehlern im Umweltrecht, 2013, S. 173 ff. m. w. N.
} 
im Lichte des Art. 9 Abs. 3 AK und der Spruchpraxis des ACCC eine subjektive Rechtsposition der Umweltverbände abgeleitet hat, aufgrund derer die Verbände im Wege der Verletztenklage die Aufstellung eines den zwingenden Vorschriften des Luftqualitätsrechts entsprechenden Luftreinhalteplans verlangen könnten. ${ }^{15}$ Die eher gezwungen wirkenden Ausführungen des BVerwG, mit denen ein subjektives Recht der Umweltverbände begründet wird, macht deutlich, dass gesetzgeberischer Handlungsbedarf besteht: Nicht durch die richterrechtliche Ausweitung der Verletztenklage, sondern durch die gesetzgeberische Erweiterung der Umweltverbandsklage sollte eine konventionskonforme Rechtslage geschaffen werden. ${ }^{16}$

\subsubsection{Klagebefugnis ( $§ 2$ Abs. 1 UmwRG) und Begründetheitsprüfung (§ 2 Abs. 5 UmwRG)}

Nach $\S 2$ Abs. 1 Nr. 1 UmwRG muss der Umweltverband geltend machen, dass die angegriffene Entscheidung Rechtsvorschriften verletzt, die dem Umweltschutz dienen und für die Entscheidung von Bedeutung sein können. Mit dieser Regelung über die Klagebefugnis korreliert $§ 2$ Abs. 5 Satz 1 Nr. 1 UmwRG, der die Begründetheit der Klage an denselben qualifizierten Rechtsverstoß knüpft.

Diese Beschränkung der Klagebefugnis und der Begründetheitsprüfung ist ebenfalls vom ACCC beanstandet worden. ${ }^{17}$ Zwar sei die Eingrenzung der rügefähigen Rechtsvorschriften auf solche, die für die Entscheidung von Bedeutung sein können, grundsätzlich zulässig. ${ }^{18}$ Dass die verletzten Rechtsvorschriften dem Umweltschutz dienen müssten, beschränke die Umweltverbandsklage allerdings in einer Weise, die nicht in Art. 9 Abs. 2 AK vorgesehen sei. ${ }^{19}$ Zwar müssten die Vertragsparteien den Konventionstext nicht wörtlich in ihr Recht übernehmen, aber sie dürften auch keine zusätzlichen Hürden aufstellen, für die es in der Konvention keine rechtliche Grundlage gebe. ${ }^{20}$ Im Gegensatz zu Art. 9 Abs. 3 AK beschränkt Absatz 2 den Umfang der Rechtmäßigkeitskontrolle gerade nicht auf „umweltbezogene Bestimmungen“, sondern spricht ohne Einschränkungen von der „materiellrechtliche[n] und verfahrensrechtliche[n] Rechtsmäßigkeit von Entscheidungen“", verlangt also nach einer umfassenden Rechtmäßigkeitskontrolle im Rahmen der

\footnotetext{
${ }^{15}$ BVerwG, Urt. v. 5.9.2013 - 7 C 21.12, NuR 2014, 37, 40 ff.

${ }^{16}$ Greim, BayVBl. 2014, 517 (521).

${ }^{17}$ Ausf. dazu Berkemann, DVBl. 2015, 389 ff.

${ }_{18}$ ACCC/C/2008/31 (Germany), Tz. 87.

${ }^{19}$ ACCC/C/2008/31 (Germany), Tz. 76.

${ }^{20} \mathrm{ACCC} / \mathrm{C} / 2008 / 31$ (Germany), Tz. $77 \mathrm{f}$.
} 
Umweltverbandsklage, mit der eine Beschränkung der Klagebefugnis und der Begründetheitsprüfung auf Rechtsvorschriften, die dem Umweltschutz dienen, nach Ansicht des ACCC unvereinbar ist. ${ }^{21}$ Die Vertragsstaatenkonferenz hat diese Einschätzung bestätigt und Deutschland empfohlen, sicherzustellen, dass Umweltverbände sowohl die materiell- als auch die verfahrensrechtliche Rechtmäßigkeit einer Maßnahme i. S. v. Art. 6 AK überprüfen lassen können, ohne geltend machen zu müssen, dass die angegriffene Entscheidung Rechtsvorschriften verletzt, die dem Umweltschutz dienen. ${ }^{22}$

\subsubsection{Präklusion (§ 2 Abs. 3 UmwRG)}

Europarechtlicher Anpassungsdruck ergibt sich mit Blick auf die Präklusionsvorschrift des § 2 Abs. 3 UmwRG, wonach der Umweltverband im Rahmen der Verbandsklage mit allen Einwendungen ausgeschlossen ist, die sie im Entscheidungsverfahren, bei dem sie Gelegenheit zur Äußerung gehabt hat, nicht rechtzeitig geltend gemacht hat, aber hätte geltend machen können. Der EuGH hat mit Urteil vom 15.10.2015 im Vertragsverletzungsverfahren gegen Deutschland entschieden, dass diese Präklusionregelung (sowie § 73 Abs. 4 VwVfG) mit Art. 11 UVP-RL und Art. 25 IE-RL unvereinbar ist. ${ }^{23}$ Die Richtlinienbestimmungen forderten einen möglichst weitreichenden Gerichtszugang sowie eine umfassende materiellrechtliche und verfahrensmäßige Kontrolle der angefochtenen Entscheidung ein, womit eine Präklusion nicht - auch nicht unter den Gesichtspunkten der Rechtssicherheit und Verfahrenseffizienz - vereinbar sei. ${ }^{24}$ Aufgrund des Vorrangs des Unionsrechts ist $\S 2$ Abs. 3 UmwRG bereits ohne gesetzgeberisches Einschreiten jedenfalls mit Blick auf Rechtsvorschriften mit unionsrechtlichem Hintergrund unanwendbar. ${ }^{25}$

\footnotetext{
${ }^{21}$ Anders vor der Entscheidung des ACCC noch BVerwG, Urt. v. 24.10.2013 - C 7 36.11, NuR 2014, 199, 200, das bemüht war, mit systematischen und teleologischen Argumenten die Vorgaben des Art. 9 Abs. 2 AK zu relativieren und noch nicht einmal eine Vorlage an den EuGH formulierte; sehr krit. dazu Berkemann, DVBl. 2015, 389, 392 ff.

22 V/9h ECE/MP.PP/2014/CRP.4, S. 1.

${ }^{23}$ EuGH, Urt. v. 15.10.2015 - C-137/14, ECLI:EU:C:2015:683, NuR 2015, 765 m. Anm. Frenz, NuR 2015, $832 \mathrm{ff}$.

${ }^{24}$ EuGH, Urt. v. 15.10.2015 - C-137/14, ECLI:EU:C:2015:683, Rdnr. 75 ff., NuR 2015, 765, 771.

${ }^{25}$ Frenz, NuR 2015, 832, 833 f. geht davon aus, dass die Präklusion auch darüber hinaus entfällt, da der Gesetzgeber auch rein nationale Vorschriften in den Bereich der UVP und damit in den Anwendungsbereich des UmwRG einbezogen habe; eine "gespaltene" Anwendung des $\S 2$ Abs. 3 UmwRG ist allerdings ebenso vorstellbar und dürfte dem gesetzgeberischen Willen besser entsprechen.
} 


\subsubsection{Fehlerfolgen ( $\$ 4$ Abs. 1 UmwRG)}

Dem europarechtlichen Anpassungsdruck im Bereich der Fehlerfolgen ${ }^{26}$ hat der Gesetzgeber bereits mit dem Änderungsgesetz vom 20.11.2015 nachgegeben, ${ }^{27}$ das $\S 4$ UmwRG an die Vorgaben des EuGH anpasst. Zweifel an der Unionsrechtskonformität des Fehlerfolgenregimes im Übrigen, insb. des $§ 4$ Abs. 2 UmwRG (Anwendung der $\S \S 214$ f. BauGB bei UVP-pflichtigen Bauleitplänen) haben sich bislang nicht erhärtet. ${ }^{28}$

\subsection{5 Überleitungsvorschriften (§5 UmwRG)}

Auch die vom EuGH wiederholt beanstandete ${ }^{29}$ Überleitungsvorschrift des $\S 5$ UmwRG wurde vom Gesetzgeber ebenfalls mit dem Änderungsgesetz 2015 an die europarechtlichen Vorgaben angepasst.

\section{Gesetzentwurf der Bundesregierung}

Mit Kabinettsbeschluss vom 22. 6. 2016 hat die Bundesregierung, basierend auf einem Referentenentwurf des BMUB, ${ }^{30}$ den Entwurf eines Gesetzes zur Anpassung des UmwRG und anderer Vorschriften an europa- und völkerrechtliche Vorgaben (RegE) verabschiedet. ${ }^{31}$ Erklärte Zielsetzung des RegE ist es, die europa- und völkerrechtlichen Vorgaben „im Wege einer 1:1-Umsetzung“ im deutschen Recht zu implementieren. ${ }^{32}$ Bereits am 27. 4.2016 beschloss das Kabinett den Gesetzentwurf zur Umsetzung der Seveso-III-RL, ${ }^{33}$ mit dem $\S 1$ Abs. 1 Satz 1 UmwRG um die Nummern 2a und 2b ergänzt werden soll. Dadurch wird die Zulassung

\footnotetext{
${ }^{26}$ EuGH, Urt. v. 7.11.2013 - C-72/12, ECLI:EU:C:2013:712, Rdnr. 36 ff. NuR 2013, 878, 881 ff. wiederholt in EuGH, Urt. v. 15.10.2015 - C-137/14, ECLI:EU:C:2015:683, Rdnr. 47 ff., NuR 2015, 765, 769.

${ }^{27}$ Gesetz zur Änderung des Umwelt-Rechtsbehelfsgesetzes zur Umsetzung des Urteils des Europäischen Gerichtshofs vom 7.11.2013 in der Rechtssache C-72/12 vom 20. 11. 2015, BGBl. I, S. 2069. ${ }_{28}$ Vgl. aber Berkemann, DVBl. 2016, 205 (211) bezugnehmend u. a. auf Kment, DVBl. 2007, 1275 ff.

${ }^{29}$ EuGH, Urt. v. 7.11.2013 - C 72/12, ECLI:EU:C:2013:712, NuR 2013, 878; Urt. v. 15.10.2015 - C-137/14, ECLI:EU:C:2015:683, NuR 2015, 765.

${ }^{30}$ Abrufbar unter

http://www.bmub.bund.de/fileadmin/Daten_BMU/Download_PDF/Gesetze/entwurf_umwRG_bf.pdf; zuletzt abgerufen am 18.7.2016.

${ }^{31}$ Abrufbar unter

http://www.bmub.bund.de/fileadmin/Daten_BMU/Download_PDF/Strategien_Bilanzen_Gesetze/um weltrechtsbehelfsgesetz_entwurf_bf.pdf; zuletzt abgerufen am 18.7.2016.

${ }^{32}$ RegE, S. 2

${ }^{33}$ Entwurf eines Gesetzes zur Umsetzung der Richtlinie 2012/18/EU zur Beherrschung der Gefahren schwerer Unfälle mit gefährlichen Stoffen, zur Änderung und anschließenden Aufhebung der Richtlinie 96/82/EG des Rates, abrufbar unter http://www.bmub.bund.de/fileadmin/Daten_BMU/Download_PDF/Chemikaliensicherheit/seveso_ric htlinie_gesetzentwurf_bf.pdf; zuletzt abgerufen am 18.7.2016.
} 
störfallrechtlich relevanter Anlagen, soweit sie noch nicht von $\S 1$ Abs. 1 Satz 1 Nr. 2 UmwRG erfasst ist, in den Anwendungsbereich der Umweltverbandsklage einbezogen. ${ }^{34}$ Der hier besprochene RegE knüpft an diese Änderungen an.

\subsection{Erweiterung des Anwendungsbereichs (§ 1 Abs. 1 Satz 1 Nr. 4 bis 6 UmwRG-E)}

Vorgesehen ist zunächst eine Erweiterung des Anwendungsbereichs der Umweltverbandsklage durch drei neue Nummern in $\S 1$ Abs. 1 Satz 1 UmwRG.

\subsubsection{Potentiell SUP-pflichtige Pläne und Programme (Nr. 4)}

Nach der neuen Nummer 4 erfasst das UmwRG nun auch Entscheidungen über die Annahme (vgl. § 141 UVPG) von Plänen und Programmen i. S. d. § 2 Abs. 5 UVPG, wenn für diese nach Anl. 3 zum UVPG oder nach landesrechtlichen Vorschriften eine Pflicht zur SUP bestehen kann. Erfasst werden damit Pläne und Programme, für die die SUP bereits zwingend vorgeschrieben ist (insb. nach $\S \S 14 b$ Abs. 1, 14c UVPG), und solche, bei denen sich aus einer SUP-Vorprüfung die SUP-Pflicht ergeben kann (insb. nach §§ 14b Abs. 2 und 4, 14d UVPG). ${ }^{35}$ Der RegE ist bemüht zu betonen, dass damit zwar ein neuer Angriffsgegenstand für die Umweltverbandsklage anerkannt wird, dass man diesen aber nicht dem weiten Gerichtszugang des Art. 9 Abs. 2 AK unterwerfen wolle - was die Vorschrift ja ausdrücklich zulässt -, sondern nur den Anforderungen des Art. 9 Abs. 3 AK Rechnung trage, die nach der Spruchpraxis des ACCC z. B. auch für Bauleitpläne gälten. ${ }^{36}$ Gleichzeitig werde damit das Urteil des BVerwG zur Klagebefugnis der Umweltverbände mit Blick auf Luftreinhaltepläne aufgegriffen. ${ }^{37}$

Ausgenommen sind nach Halbsatz 2 Pläne und Programme, über deren Annahme durch formelles Gesetz entschieden wird. Im Bundesrecht kann dies Verkehrswegeplanungen, energiewirtschaftliche Bundesbedarfspläne und Festlegungen nach dem Standortauswahlgesetz betreffen. ${ }^{38}$ Diese Ausnahme wird auf Art. 9 Abs. 3 i. V. m. Art. 2 Nr. 2 AK gestützt, wonach Gesetzgebungsorgane nicht unter den für den Gerichtszugang relevanten Behördenbegriff fallen. ${ }^{39}$

\footnotetext{
${ }^{34}$ Dadurch wird Art. 23 lit. b RL 2012/18/EU = Seveso-III-RL umgesetzt, der vergleichbar Art. 11 UVPRL und Art. 25 IE-RL den Zugang zu den Gerichten regelt; die erfassten Verfahren fallen in den Bereich des Art. 9 Abs. 2 AK.

${ }^{35} \operatorname{RegE}$, S. 31.

${ }^{36} \operatorname{RegE}$, S. 32.

${ }^{37} \operatorname{RegE}$, S. 33.

${ }^{38}$ RegE, S. 33 bezugnehmend auf Nr. 1.1, 1.10, 1.15 und 1.16 Anl. 3 zum UVPG.

${ }^{39} \operatorname{RegE}$, S. 33.
} 


\subsubsection{Zulassung sonstiger Vorhaben (Nr. 5)}

Die neue Nummer 5 soll die Zulassung sonstiger Vorhaben, d. h. solche, die nicht schon unter die Nummern 1 bis $2 b$ fallen, durch Verwaltungsakt oder öffentlichrechtlichen Vertrag erfassen. Voraussetzung ist aber, dass diese Vorhaben unter Anwendung umweltbezogener Rechtsvorschriften des Bundesrechts, des Landesrechts oder unmittelbar geltender Rechtsakte der EU zugelassen werden.

Nach dem RegE orientiert sich der Vorhabenbegriff an § 2 Abs. 2 UVPG, allerdings ohne den Verweis auf Anl. 1 zum UVPG. Erfasst sind also Errichtung und Betrieb von technischen Anlagen, der Bau von sonstigen Anlagen, die Durchführung einer sonstigen in Natur und Landschaft eingreifenden Maßnahme sowie jeweils Änderungen und Erweiterungen. ${ }^{40}$

Das Vorhaben muss durch Verwaltungsakt oder öffentlich-rechtlichen Vertrag zugelassen werden, wobei unter den Begriff der Zulassung auch besondere Instrumente wie Teilgenehmigungen oder Vorbescheide fallen sollen. ${ }^{41}$

Schließlich müssen bei der Zulassung umweltbezogene Rechtsvorschriften anzuwenden sein. Entscheidend ist nicht - auch wenn der Wortlaut hier nicht ganz eindeutig ist -, dass diese Rechtsvorschriften von der Behörde auch angewendet wurden; ihre Anwendbarkeit genügt. ${ }^{42}$ Der Begriff der umweltbezogenen Rechtsvorschriften ist aus der (nicht authentischen) deutschen Fassung von Art. 9 Abs. 3 AK übernommen („,umweltbezogene Bestimmungen des innerstaatlichen Rechts“). Der Begriff wird in 1 Abs. 4 UmwRG-E legaldefiniert (dazu sogleich).

Neben Bundes- und Landesrecht kommt das unmittelbar geltende Recht der EU als Rechtsquelle in Betracht, d. h. alle EU-Rechtsakte, die nicht erst durch mitgliedstaatliche Umsetzung Geltung erlangen. Der RegE bezieht sich neben Verordnungen nach Art. 288 Abs. 2 AEUV auch auf die Rechtsprechung des EuGH zur unmittelbaren Geltung von Richtlinien im Falle fehlender oder fehlerhafter Umsetzung. ${ }^{43}$

\subsection{3 Überwachungs- und Aufsichtsmaßnahmen (Nr. 6)}

\footnotetext{
40 RegE, S. 33.

41 RegE, S. 33.

${ }^{42}$ Vgl. RegE, S. 33: „Maßgeblich [...] ist jeweils allein, ob für die Zulassungsentscheidung umweltbezogene Vorschriften [...] anzuwenden sind."

${ }^{43}$ RegE, S. 34.
} 
Als letzte Kategorie werden in Nummer 6 Verwaltungsakte über Überwachungsoder Ausführungsmaßnahmen zur Umsetzung oder Durchführung von Entscheidungen nach den Nummern 1 bis 5 genannt, die der Einhaltung umweltbezogener Rechtsvorschriften des Bundesrechts, Landesrechts oder unmittelbar geltender EU-Rechtsakte dienen.

Der RegE will damit den Vorgaben der AK-Institutionen genügen, die explizit das Fehlen von Verbandsklagemöglichkeiten gegen die Anwendung umweltbezogener Bestimmungen durch Behörden und Private über den Kreis der Zulassungsentscheidungen hinaus beanstandet hatten. ${ }^{44}$ In der Entwurfsbegründung wird die deutsche Verwaltungsrechtstradition betont, nach der nur Rechtsbehelfe gegen eine Behörde (und nicht gegen den Privaten selbst) in Betracht kämen. ${ }^{45}$ Als Angriffsgegenstand werden daher Überwachungs- und Ausführungsverwaltungsakte genannt, die an die Privaten adressiert sind und diese zur Einhaltung ihrer umweltbezogenen Rechtspflichten anhalten. Es geht also um Verpflichtungsklagen der Umweltverbände auf die Vornahme von Aufsichtsmaßnahmen, die einen umweltrechtskonformen Zustand, d. h. eine Übereinstimmung mit „,umweltbezogenen Rechtsvorschriften“ i. S. v. § 1 Abs. 4 UmwRG-E, herstellen sollen. ${ }^{46}$ Auf die Vornahme behördeninterner Maßnahmen ohne Verwaltungsaktqualität (z. B. Weisungen im Rahmen der Bundesauftragsverwaltung) sollen Umweltverbände dagegen nicht klagen können. ${ }^{47}$

\subsubsection{Sonderregelungen (§ 1 Abs. 1 Satz 3 UmwRG-E)}

Der RegE ergänzt die Auflistung der Sondervorschriften in Satz 3 (derzeit $\S \S 15$ Abs. 5, 16 Abs. 3 UVPG und $\S 44 a$ VwGO) um $\S 17$ Abs. 4 Sätze 3 bis 5 StandAG, $\S 15$ Abs. 3 Satz 2 NABEG und $\S 17$ a Abs. 5 Satz 1 EnWG sowie „andere entsprechende Rechtsvorschriften“. Rechtsvorschriften entsprechen den aufgelisteten, wenn sie den Anwendungsbereich des UmwRG modifizieren, insb. einschränken. Zu den entsprechenden Rechtsvorschriften gehören nach der Begründung die im RegE ebenfalls vorgesehenen $\S \S 16$ Abs. 4 UVPG. 19b Abs. 2 UVPG, die bestimmte Raumordnungspläne für die Windenergie und den Rohstoffabbau sowie die Bundesverkehrswegeplanung aus $\S 1$ Abs. 1 Satz 1 Nr. 4 UmwRG ausnehmen.

\footnotetext{
${ }^{44}$ ACCC/C/2008/31 (Germany), Tz. 100; V/9h ECE/MP.PP/2014/CRP.4, S. 2.

45 RegE, S. 34.

46 Vgl. RegE, S. 34.

${ }^{47}$ RegE, S. 34.
} 


\subsection{Legaldefinition der umweltbezogenen Rechtsvorschriften (§ 1 Abs. 4 UmwRG- E)}

Ein Schlüsselbegriff des RegE sind die „umweltbezogenen Rechtsvorschriften“. Nicht nur die Tatbestände des $\S 1$ Abs. 1 Satz 1 Nr. 5 und 6 knüpfen an ihn an. Er findet auch Verwendung in den Vorschriften über die Zulässigkeit (§ 2 Abs. 1 Satz 2 UmwRG-E) und Begründetheit (§ 2 Abs. 4 Nr. 2 UmwRG-E) der Umweltverbandsklage.

Nach der Legaldefinition des $\S 1$ Abs. 4 UmwRG-E sind umweltbezogene Rechtsvorschriften Bestimmungen, die sich zum Schutz von Mensch und Umwelt auf (Nr. 1) den Zustand von Umweltbestandteilen i. S. v. § 2 Abs. 3 Nr. 1 UIG oder (Nr. 2) Faktoren i. S. v. $§ 2$ Abs. 3 Nr. 2 UIG beziehen. Maßgeblich ist also nicht der unbestimmte Rechtsbegriff "Umweltschutz", der in der Vergangenheit zu unterschiedlichen Deutungen Anlass gab. ${ }^{48}$ Der RegE knüpft vielmehr an die bestehenden Begriffsbestimmungen in § 2 Abs. 3 UIG an, der immerhin Art. 2 Nr. 3 AK („Informationen über die Umwelt“") wörtlich wiedergibt.

Der RegE verweist zudem für „weitergehende Prüfungen“ auf die Spruchpraxis des ACCC. ${ }^{49}$ Man wird dies als einen Appell an die Gerichte verstehen können, deren Entscheidungen bislang eine vertiefte Auseinandersetzung mit den Ansichten der AK-Institutionen oftmals haben vermissen lassen. ${ }^{50}$ Jedenfalls der unmittelbare normative Bezug zum Völkerrecht ist damit - so der RegE denn Gesetz wird - kraft gesetzgeberischer Anordnung hergestellt.

\subsection{Klagebefugnis (§ 2 Abs. 1 UmwRG-E)}

In deutlicher Abkehr von der bisherigen Rechtslage sieht der RegE in § 2 Abs. 1 UmwRG-E eine nach Anfechtungsgegenständen differenzierende Regelung der Klagebefugnis vor. Unverändert bleibt lediglich Nummer 2, wonach der Umweltverband geltend machen muss, durch die angegriffene Entscheidung in seinem satzungsgemäßen Aufgabenbereich der Förderung der Ziele des Umweltschutzes berührt zu sein.

\subsubsection{Klagebefugnis in den Fällen der $\S \S 1$ Abs. 1 Satz 1 Nr. 1 und 2 UmwRG}

\footnotetext{
${ }^{48}$ Vgl. Bunge, UmwRG, 2013, § 2 Rdnr. 28 ff.

${ }^{49}$ RegE, S. 34.

${ }^{50}$ Berkemann, DVB1. 2015, 389, 399.
} 
In den Fällen, für die bereits bislang das UmwRG galt, d. h. im Anwendungsbereich der UVP- und IE-RL muss der Umweltverband geltend machen, dass die angegriffene Entscheidung oder deren Unterlassen Rechtsvorschriften widerspricht, die für die Entscheidung von Bedeutung sein können (Nr. 1). Der Kreis der rügefähigen Rechtsvorschriften wird nicht weiter eingegrenzt. Die Beschränkung auf Rechtsvorschriften, die dem Umweltschutz dienen, entfällt im Einklang mit Art. 9 Abs. 2 AK.

Außerdem muss der Umweltverband im Verfahren zur Beteiligung berechtigt gewesen sein (Nr. 3 Buchst. a). Anders als nach der bisherigen Regelung muss er sich dagegen nicht in der Sache geäußert bzw. keine Gelegenheit zur Äußerung gehabt haben. Damit soll dem EuGH-Urteil vom 15. 10. 2015 zur Präklusion entsprochen werden. ${ }^{51}$

\subsubsection{Klagebefugnis in den Fällen der $\S \S 1$ Abs. 1 Satz 1 Nr. 2a bis 6 UmwRG}

Für die Gegenstände der Umweltverbandsklage, die außerhalb des Anwendungsbereichs der UVP- und IE-RL und des dahinterstehenden Art. 9 Abs. 2 AK liegen, stellt der RegE andere Anforderungen an die Klagebefugnis.

\subsubsection{Verletzung umweltbezogener Rechtsvorschriften}

$\S 2$ Abs. 1 Satz 2 UmwRG-E verlangt, dass der Verband zudem die Verletzung umweltbezogener Rechtsvorschriften geltend macht. Diese Beschränkung des Kreises rügefähiger Rechtsvorschriften stützt sich auf die entsprechende Formulierung in Art. 9 Abs. 3 AK. ${ }^{52}$ Satz 2 muss mit Satz 1 Nr. 1 im Zusammenhang gelesen werden: Der Umweltverband muss geltend machen, dass die angegriffene Entscheidung umweltbezogenen Rechtsvorschriften widerspricht, die für die Entscheidung von Bedeutung sein können. Rügt er hingegen nur Verstöße gegen Bestimmungen, die nicht unter den durch $\S 1$ Abs. 4 UmwRG-E definierten Begriff der umweltbezogenen Rechtsvorschriften fallen, ist die Klage unzulässig.

\subsubsection{Verfahrensbeteiligung}

Was die vorherige Verfahrensbeteiligung anbelangt, stellt Satz 1 Nr. 3 für die neuen Klagegegenstände unterschiedliche Anforderungen auf:

Für Entscheidungen, die unter die Seveso-III-Richtlinie fallen (§ 1 Abs. 1 Satz 1 Nr. 2a und 2b UmwRG-E), gilt Satz 1 Nr. 3 Buchst. a, d. h., der Verband muss nur zur Beteiligung berechtigt gewesen sein. Zwar sind diese Entscheidungen nicht von

\footnotetext{
51 RegE, S. 36.

52 RegE, S. 36.
} 
Art. 9 Abs. 2 AK erfasst, doch erklärt Art. 23 Buchst. b Seveso-III-RL den Art. 11 UVP-RL, zu dem sich der EuGH geäußert hatte, für entsprechend anwendbar, so dass auch insoweit keine Präklusionsvorschrift vorgesehen werden konnte.

Für Entscheidungen über potentiell SUP-pflichtige Pläne und Programme (§ 1 Abs. 1 Satz 1 Nr. 4 UmwRG-E) wird dagegen in Satz 1 Nr. 3 Buchst. b an der bisherigen Regelung festgehalten, wonach der Verband zur Verfahrensbeteiligung berechtigt sein, sich in der Sache geäußert haben bzw. keine Gelegenheit zur Äußerung gehabt haben musste. ${ }^{53}$

In den sonstigen Fällen ( $§ 1$ Abs. 1 Nr. 3, 5 und 6 UmwRG-E) muss der Verband weder zur Beteiligung berechtigt gewesen sein noch sich am Verfahren beteiligt haben.

\subsection{Begründetheit ( 2 Abs. 4 UmwRG-E)}

Die neue Vorschrift über die Begründetheit der Klage findet sich in $\S 2$ Abs. 4 UmwRG-E. Es wird hier die in der Klagebefugnis vorgenommene Differenzierung zwischen den bisherigen und den neuen Anfechtungsgegenständen, d. h. zwischen Art. 9 Abs. 2 und Abs. 3 AK fortgeführt: In den Fällen der $\S 1$ Abs. 1 Satz 1 Nr. 1 und 2 UmwRG ist die Klage begründet, soweit die Entscheidung oder deren Unterlassen gegen Rechtsvorschriften verstößt, die für sie von Bedeutung sind (Satz 1 Nr. 1). Alle anderen Entscheidungen müssen gegen umweltbezogene Rechtsvorschriften verstoßen (Satz 1 Nr. 2).

Einschränkend hält der RegE in beiden Varianten daran fest, dass der Verstoß Belange berühren muss, die zu den Zielen gehören, die der Umweltverband nach seiner Satzung fördert (Satz 1 a. E.). Damit soll „im Ergebnis“ verhindert werden, dass im Rahmen der Umweltverbandsklage jeglicher Rechtsverstoß gerügt werden kann. ${ }^{54}$ Im Gegensatz zur bisherigen Regelung - und dem unveränderten $§ 2$ Abs. 1 Nr. 2 UmwRG - spricht der RegE hier nicht mehr von „Belangen des Umweltschutzes“, sondern schlicht von „Belangen“. Ob damit eine bestimmte Regelungsabsicht zum Ausdruck kommt, erhellt aus der Entwurfsbegründung nicht.

\footnotetext{
${ }^{53}$ Vgl. RegE, S. 36.

54 RegE, S. 36.
} 
Dort wird lediglich betont, dass die Einschränkung des gerichtlichen Kontrollumfangs im Einklang mit der AK stehe. ${ }^{55}$

Mit Blick auf $\S 1$ Abs. 1 Satz 1 Nr. 1 und Nr. 4 UmwRG-E, wo an das potentielle Bestehen einer UVP/SUP-Pflicht angeknüpft wird, bestimmt § 2 Abs. 4 Satz 2 UmwRG-E, dass die Klage nur begründet ist, wenn eine Pflicht zur Durchführung einer Umweltprüfung i. S. v. § 1 Nr. 1 UVPG (= UVP und SUP) bestand. Nach dem RegE hat das Gericht dies als ersten Punkt im Rahmen der Begründetheit zu prüfen. Erweist sich das Vorhaben weder als UVP- noch als SUP-pflichtig, ist die Klage ohne weiteres unbegründet. ${ }^{56}$

\subsection{Präklusion}

Der Einwendungsausschluss nach § 2 Abs. 3 UmwRG fällt vollständig weg. Der RegE trägt damit der EuGH-Entscheidung vom 15. 10. 2015 Rechnung. Außerdem bestimmt $\S 7$ Abs. 4 UmwRG-E, dass in Verfahren gegen eine Entscheidung nach $\S 1$ Abs. 1 Satz 1 Nr. 1 bis 2b UmwRG-E, d. h. bei Vorhaben, die unter die UVP-, IE-, oder Seveso-III-RL fallen, die allgemeine planfeststellungsrechtliche Präklusionsregelung des $\S 73$ Abs. 4 Satz 3 bis 6 VwVfG keine Anwendung findet.

Sofern sie einschlägig sind, gelten die sonstigen Präklusionsvorschriften bei den anderen Klagegegenständen allerdings fort. ${ }^{57}$

Für potentiell SUP-pflichtige Pläne und Programme (§ 1 Abs. 1 Satz 1 Nr. 4 UmwRGE) gilt das Erfordernis, dass sich der Verband, sofern ihm Gelegenheit gegeben wurde, überhaupt in der Sache geäußert hat (§ 2 Abs. 1 Satz 1 Nr. 3 Buchst. b UmwRG-E); anderenfalls ist die Klage bereits unzulässig. Darüber hinaus sieht $\S 7$ Abs. 3 Satz 1 UmwRG-E eine materielle Einwendungspräklusion entsprechend dem bisherigen $\S 2$ Abs. 3 UmwRG für Klagen gegen diese Pläne und Programme vor. Eine praktisch wichtige Ausnahme ordnet Satz 2 für Bebauungspläne an. Der RegE will durch diese pauschale Ausnahme die (komplizierte) Prüfung entbehrlich machen, ob ein SUP-pflichtiger Bebauungsplan zugleich UVP-pflichtig ist. ${ }^{58}$

Für sonstige Zulassungsentscheidungen ( 11 Abs. 1 Satz 1 Nr. 5 UmwRG-E), sofern sie als Planfeststellungsbeschlüsse ergehen, gilt weiterhin die materielle Einwendungspräklusion des $§ 74$ Abs. 4 Satz 3 bis 5 VwVfG.

\footnotetext{
55 RegE, S. 36.

${ }^{56}$ Vgl. RegE, S. 31/36.

57 Vgl. RegE, S. 41.

58 RegE, S. 41.
} 
Über das UmwRG hinaus modifiziert der RegE zudem die Präklusionsregelungen im bestehenden Recht: In § 9 Abs. 1c UVPG-E soll nunmehr eine Präklusion von Einwendungen für das Zulassungsverfahren vorsehen, sofern die Einwendungen für die Rechtmäßigkeit der Entscheidung nicht von Bedeutung sind. Der Einwendungsausschluss in $\S 10$ Abs. 3 Satz 5 BImSchG wird auf das Genehmigungsverfahren beschränkt, führt also nicht mehr zu einer im gerichtlichen Verfahren beachtlichen materiellen Präklusion. § 47 Abs. 2a VwGO soll aufgehoben werden.

Eine materielle Einwendungspräklusion kommt nach dem RegE also nur noch in Betracht bei SUP-pflichtigen Plänen und Programmen mit Ausnahme der Bebauungspläne sowie bei sonstigen Vorhaben i. S. d. § 1 Abs. 1 Satz 1 Nr. 5 UmwRG-E, die durch Planfeststellungsbeschluss zugelassen werden. Alle anderen materiellen Präklusionstatbestände sollen entfallen.

\subsection{Einwendungsausschluss bei Missbrauch oder unredlichem Verhalten ( 5 UmwRG-E)}

Mit § 5 UmwRG-E will der RegE den geringen Spielraum nutzen, den der EuGH in seinem Urteil vom 15.10.2015 dem mitgliedstaatlichen Gesetzgeber zur Begrenzung der Einwendungen noch zugestanden hat. ${ }^{59}$ Nachdem er die bisherigen Präklusionsvorschriften für richtlinienwidrig erklärt hat, führt der EuGH nämlich aus, dass „spezifische Verfahrensvorschriften“ möglich seien, „nach denen z. B. ein missbräuchliches oder unredliches Vorbringen unzulässig ist", wenn diese „geeignete Maßnahmen darstellen, um die Wirksamkeit des gerichtlichen Verfahrens zu gewährleisten. "60 Diese Passage greift § 5 UmwRG-E beinahe wörtlich auf:

Einwendungen, die erstmals im Rechtsbehelfsverfahren erhoben werden, bleiben unberücksichtigt, wenn die erstmalige Geltendmachung im Rechtsbehelfsverfahren missbräuchlich oder unredlich ist.

Die Entwurfsbegründung stellt zunächst klar, dass der Kläger bereits im Verwaltungsverfahren die Gelegenheit gehabt haben muss, sich zu äußern. Unterbleibt diese Äußerung und stellt sich das erstmalige Vorbringen im Rechtsbehelfsverfahren dem Gericht im Einzelfall als missbräuchlich oder unredlich dar, ist es zurückzuweisen. ${ }^{61}$ Missbräuchlich oder unredlich könne das Vorbringen beispielsweise dann sein, wenn im Verwaltungsverfahren erklärt oder auf andere

\footnotetext{
${ }^{59} \mathrm{Vgl}$. RegE, S. 38.

${ }^{60}$ EuGH, Urt. v. 15.10.2015 - C.137/14, ECLI:EU:C:2015:683, Rdnr. 81, NuR 2015, 765, 771.

${ }^{61} \mathrm{Vgl}$. RegE, S. 38.
} 
Weise deutlich gemacht wurde, dass entsprechende Einwendungen nicht bestünden. Bei Umweltverbänden könne missbräuchliches oder unredliches Verhalten etwa dann vorliegen, wenn die erstmalige Erhebung von Einwendungen, die dem Verband bereits im Zulassungsverfahren bekannt gewesen seien, den Schutzanliegen und Umweltbelangen, als deren Sachwalter sich die Vereinigung verstehe, zuwiderlaufe, die Vereinigung sich also gemessen an den Zielen ihrer Satzung oder ihrer Rolle als „Quasi-Verwaltungshelfer" ${ }^{\text {" }}$,unvernünftig“ verhalte. ${ }^{63}$

\subsection{Sonstige Änderungen im Überblick}

In § 3 Abs. 1 Satz 5 UmwRG-E wird die Veröffentlichung der Anerkennung einer Vereinigung im Internet vorgeschrieben, um den Vorhabenträgern einen Überblick über die anerkannten Verbände zu verschaffen. ${ }^{64} \S 4$ UmwRG-E (Verfahrensfehler) wird um die Seveso-III-Tatbestände ergänzt. Außerdem wird Absatz 3 klarstellend überarbeitet und die Vorschrift um zwei neue Absätze ergänzt. Absatz 4 Satz 1 erklärt die Unterscheidung zwischen absoluten und relativen Verfahrensfehlern in Absatz 1 bei SUP-pflichtigen Plänen und Programmen für entsprechend anwendbar. Nach Satz 2 gelten für Raumordnungspläne stattdessen jedoch die §§ 12, 28 Abs. 2 ROG bzw. die einschlägigen landesrechtlichen Vorschriften. Absatz 5 schließt die Anwendung des $\S 4$ Abs. 1 UmwRG auf die anderen neuen Klagegegenstände aus, indem er auf die fachrechtlichen bzw. allgemeinen verwaltungsverfahrensrechtlichen Regelungen verweist.

Der bisherige § 4a UmwRG (Maßgaben zur Anwendung der VwGO) soll entfallen. Damit erübrigen sich auch die Bedenken gegen die Modifikation des einstweiligen Rechtsschutzverfahrens in $\S 4$ a Abs. 3 UmwRG. An der besonderen Klagebegründungsfrist wird allerdings in § 6 UmwRG-E festgehalten. ${ }^{65}$

§ 7 UmwRG-E enthält neben den bereits erwähnten Präklusionsvorschriften weitere besondere Bestimmungen für einzelne Klagegegenstände nach § 1 Abs. 1 Satz 1 UmwRG-E: $\S 7$ Abs. 1 UmwRG-E ermöglicht eine Bekanntmachung von Entscheidungen nach $\S 1$ Abs. 1 Satz 1 Nr. 5 und 6 UmwRG-E an bestimmte Personen und Vereinigungen, wenn eine öffentliche Bekanntmachung nicht vorgeschrieben ist und der Antragsteller bzw. Adressat des jeweiligen Verwaltungsakts dies beantragt. § 7 Abs. 2 Satz 1 UmwRG-E sieht vor, dass bei Klagen gegen potentiell SUP-pflichtige Pläne und Programme oder deren Unterlassen stets das OVG erstinstanzlich zuständig ist; Satz 3 regelt die örtliche

\footnotetext{
${ }^{62}$ Bezugnehmend auf BVerwG, Urt. v. 1.4.2014 - 4 C 6.14, NuR 2015, 571, 573.

${ }^{63} \operatorname{RegE}$, S. 38.

${ }^{64} \operatorname{RegE}$, S. 37.

${ }^{65} \operatorname{RegE}$, S. 39.
} 
Zuständigkeit bei länderübergreifenden Planungen. Satz 2 erklärt § 47 VwGO für entsprechend anwendbar, wenn gegen die Entscheidung weder Normenkontrollantrag noch Gestaltungs- oder Leistungsklage statthaft sind. Schließlich überträgt Absatz 5 die Heilungsmöglichkeit des $§ 75$ Abs. 1a Satz 2 VwVfG auf Entscheidungen nach $\S 1$ Abs. 1 Satz 1 Nr. 2b oder 5, d. h. Entscheidungen über die Zulässigkeit von Vorhaben innerhalb des störfallrechtlichen Sicherheitsabstands bzw. von sonstigen Vorhaben, wenn diese nicht schon durch Planfeststellungsbeschluss getroffen wird. ${ }^{66}$ Hierunter fallen $u$. a.

immissionsschutzrechtliche Genehmigungen und wasserrechtliche Erlaubnisse. ${ }^{67}$ $\S 8$ UmwRG-E enthält die Überleitungsbestimmungen. Sein Absatz 2 sieht für die neuen Klagegegenstände des $\S 1$ Abs. 1 Satz 1 Nr. 4 bis 6 UmwRG-E eine Anwendung des UmwRG vor, wenn die Entscheidungen nach dem 31.12.2016 ergangen sind oder hätten ergehen müssen.

\section{Bewertung}

Der RegE ist noch weit davon entfernt, Gesetz zu werden. Änderungen im parlamentarischen Verfahren sind möglich und - angesichts der politischen Brisanz der Ausweitung der umweltrechtlichen Verbandsklage - zu erwarten. Die vorgeschlagenen Regelungen können dennoch bereits einer Bewertung unterzogen werden. Den Maßstab sollen dabei nicht (umwelt-)rechtspolitische Wünsche, sondern allein das höherrangige Recht und die Konsistenz der gesetzgeberischen Ausgestaltung bilden.

\subsection{Ausweitung der Umweltverbandsklage}

Mit der Erweiterung der Klagegegenstände in $\S 1$ Abs. 1 Satz 1 Nr. 2a bis 6 UmwRGE erfüllt der RegE die durch Art. 9 Abs. 3 AK aufgestellten und die AK-Institutionen konkretisierten Anforderungen an den Gerichtszugang der Umweltverbände bei Maßnahmen, die gegen nationale umweltbezogene Rechtsvorschriften verstoßen. Gesetzgebungstechnisch nachvollziehbar ist, dass der RegE von einer an Art. 9 Abs. 3 AK angelehnten „Generalklausel“ abgesehen und stattdessen mit den Nummern 2a bis 6 einzelne Tatbestände geschaffen hat. Zurecht wird in der Begründung auf anderenfalls drohende Abgrenzungsschwierigkeiten hingewiesen. ${ }^{68}$ Das ACCC verlangt von den Vertragsstaaten gerade keine wörtliche Übernahme der Konvention, wohl aber dass der Gerichtszugang in solchen Fällen die Regel und nicht die Ausnahme sein sollte. ${ }^{69}$ Durch die weit gefassten Tatbestände der

\footnotetext{
${ }^{66} \operatorname{RegE}$, S. 41.

${ }^{67} \operatorname{RegE}$, S. 42.

${ }^{68}$ RegE, S. $34 \mathrm{f}$.

${ }^{69} \mathrm{Vgl}$. ACCC/C/2008/31, Tz. 92.
} 
Nummern 4 bis 6 und die Anlehnung der Begriffsbestimmung in $\S 1$ Abs. 4 UmwRGE an Art. 2 Nr. 3 AK und die Spruchpraxis des ACCC ist dieses Regel-AusnahmeVerhältnis gewährleistet.

\subsection{Klagebefugnis}

Umweltverbände müssen nun nicht mehr rügen, dass Rechtsvorschriften, die dem Umweltschutz dienen, durch die angegriffene Entscheidung verletzt werden. Es genügt zunächst die bloße Rüge eines entscheidungserheblichen Rechtsverstoßes. Damit wird in vollem Umfange den Vorgaben des Art. 9 Abs. 2 AK und der dazu ergangenen „Empfehlungen“ entsprochen. Dass bei den Entscheidungen, die nicht unter Art. 9 Abs. 2 AK fallen, einschränkend die Geltendmachung einer Verletzung umweltbezogener Rechtsvorschriften verlangt wird, ist angesichts der entsprechenden Formulierung des Art. 9 Abs. 3 AK konventionskonform.

Unbedenklich ist auch, dass $§ 2$ Abs. 1 Satz 1 Nr. 2 UmwRG-E die Klagebefugnis nach wie vor davon abhängig macht, dass der Umweltverband geltend macht, durch die Entscheidung in seinem satzungsgemäßen Aufgabenbereich der Förderung der Ziele des Umweltschutzes berührt zu sein. Das hat das ACCC ausdrücklich festgestellt. ${ }^{70}$ Mit Blick auf die - nur vermeintlich korrespondierende (s. u.) Regelung des $\S 2$ Abs. 4 Satz 1 a. E. UmwRG-E ist hervorzuheben, dass der Aufgabenbereich des Umweltverbands im Rahmen der Klagebefugnis durch die Entscheidung (möglicherweise) berührt sein muss. Es geht also stets um die Regelungswirkung der angegriffenen Entscheidung, nicht um die spezifische Rechtsvorschrift, die als verletzt gerügt wurde. ${ }^{71}$ Demnach ist etwa die Klage eines Vogelschutzverbands gegen die Zulassung einer Windenergieanlage auch dann zulässig, wenn der Verband ausschließlich die Verletzung von Rechtsvorschriften rügt, die nicht dem Vogelschutz dienen (z. B.: Lärmschutz), solange durch die zugelassene Windenergieanlage Vögel gestört oder getötet werden können. Umgekehrt könnte ein Verband, der sich satzungsmäßig dem Robbenschutz verschrieben hat, jedoch nicht gegen die Genehmigung einer Windenergieanlage in Bayern vorgehen, durch deren Errichtung und Betrieb Robben nach menschlichem

\footnotetext{
${ }^{70}$ ACCC/C/2008/31, Tz. $70 \mathrm{ff}$.

${ }^{71}$ A. A. ohne Begründung Schlacke, in: dies./Schrader/Bunge, Informationsrechte, Öffentlichkeitsbeteiligung und Rechtsschutz im Umweltrecht, 2010, § 3 Rdnr. 154; unklar Fellenberg/Schiller, in: Landmann/Rohmer, UmwR, § 2 UmwRG Rdnr. 33 ff. (Stand: April 2012), die bald auf die „verletzte Rechtsvorschrift", bald auf die „Umweltauswirkungen der angegriffenen Entscheidung“ abstellen; die hier vertretene Auffassung entspricht dem Willen des Gesetzgebers, vgl. BT-Drs. 16/2495, S. 12 r. Sp.: „Zwischen dem satzungsgemäßen Aufgabenbereich und der mit dem Rechtsbehelf angegriffenen Entscheidung muss ein Zusammenhang bestehen."
} 
Ermessen nicht in Mitleidenschaft gezogen werden können. ${ }^{72}$ Darin erschöpft sich die konventionskonforme Eingrenzung des Kreises der Klageberechtigten nach $\S 2$ Abs. 1 Satz 1 Nr. 2 UmwRG.

Soweit die präklusionsartige Regelung des § 2 Abs. 1 Nr. 3 UmwRG aufgehoben werden soll, entspricht dies den Vorgaben aus Art. 11 UVP-RL (i. V. m. Art. 23 Buchts. b Seveso-III-RL) und Art. 25 IE-RL, wie sie durch den EuGH in seinem Urteil vom 15. 10. 2015 konkretisiert wurden. Außerhalb des Anwendungsbereichs dieser Richtlinien kann daran festgehalten werden.

Etwas inkonsistent ist die Regelung der Klagebefugnis mit Blick auf Bebauungspläne. Wenn diese Zulassungsvoraussetzungen für UVP-pflichtige oder UVP-vorprüfungspflichtige Vorhaben festlegen oder Planfeststellungsbeschlüsse für solche Vorhaben ersetzen, fallen sie unter $\S 1$ Abs. 1 Satz 1 Nr. 1 UmwRG. ${ }^{73}$ Der Umweltverband kann in diesen Fällen einen Normenkontrollantrag unter den Voraussetzungen des $\S 2$ Abs. 1 Satz 1 Nr. 1 bis 3 Buchst. a UmwRG-E stellen. Er muss also weder eine Verletzung umweltbezogener Rechtsvorschriften geltend machen noch sich am Aufstellungsverfahren beteiligt haben. Ist der Bebauungsplan hingegen nur (potentiell) SUP-pflichtig, unterfällt er $\S 1$ Abs. 1 Satz 1 Nr. 4 UmwRGE und der Verband ist nur antragsbefugt, wenn er eine Verletzung umweltbezogener Rechtsvorschriften geltend macht und sich am Verfahren beteiligt hat ( $₹ 2$ Abs. 1 Satz 2, Abs. 1 Satz 1 Nr. 3 Buchst. b UmwRG-E). Es muss ggf. also bereits im Rahmen der Zulässigkeit festgestellt werden, ob der angegriffene Bebauungsplan potentiell UVP- oder lediglich SUP-pflichtig ist. Eben diese Unterscheidung will der RegE den Gerichten aber bei der materiellen Präklusionsregelung des § 7 Abs. 3 UmwRG abnehmen, indem er aus deren Anwendungsbereich Bebauungspläne explizit ausnimmt. Zurecht wird in der Begründung darauf hingewiesen, dass die Prüfung, ob ein SUP-pflichtiger Bebauungsplan zugleich einer UVP-Pflicht unterliegt, für die Praxis schwer durchführbar sei. Eben dies sei der Grund für die Einführung der einheitlichen Umweltprüfung durch das EAGBau 2004 gewesen. ${ }^{74}$ Im Rahmen der Klagebefugnis bürdet der RegE dem Rechtsanwender hingegen durch die beschriebene Ausgestaltung diese Prüfungslast auf. Diese Inkonsistenz sollte im Gesetzgebungsverfahren korrigiert werden (bspw. § 2 Abs. 1 Satz 3: „Bei Rechtsbehelfen gegen Entscheidungen über die Änderung, Ergänzung oder Aufhebung von Bebauungsplänen nach $\S 10$ des Baugesetzbuches findet ausschließlich Satz 1 Nummer 1 bis 3 Buchst. a Anwendung. “).

\footnotetext{
72 Zur Problematik der allgemein umschriebenen Satzungszwecke vgl. aber Kment, in: Hoppe/Beckmann, UVPG, 2012, § 2 UmwRG Rdnr. 10.

${ }^{73}$ Bunge, UmwRG, 2013, § 1 Rdnr. 33 ff.

${ }^{74}$ RegE, S. 41.
} 


\subsection{Begründetheit}

Der RegE ist bei der Ausgestaltung des $\S 2$ Abs. 4 UmwRG-E darum bemüht, den Prüfungsmaßstab im Rahmen der Begründetheit an die Vorgaben aus Art. 9 Abs. 2 AK anzupassen und gleichzeitig die im Rahmen von Art. 9 Abs. 3 AK zulässige Beschränkung auf umweltbezogene Rechtsvorschriften zur Geltung zu bringen.

$\mathrm{Zu}$ einer „Vollkontrolle“75 im Bereich des Art. 9 Abs. 2 AK soll es dennoch nicht kommen. Denn nach $\S 2$ Abs. 4 Satz 1 a. E. UmwRG-E wird an dem Erfordernis festgehalten, dass der Rechtsverstoß Belange berühren muss, die zu den Zielen gehören, die die Vereinigung nach ihrer Satzung fördert. Es ist zu erwarten, dass diese Einschränkung, sollte der RegE Gesetz werden, in der Gerichtspraxis mehr Aufmerksamkeit als bisher auf sich ziehen wird. Derzeit wird davon ausgegangen, dass diese Einschränkung des Kontrollmaßstabs mit dem Zulässigkeitskriterium des $\S 2$ Abs. 1 Nr. 2 UmwRG korreliert. ${ }^{76}$ Jedenfalls der Wortlaut der Bestimmungen spricht allerdings gegen einen solchen inhaltlichen Gleichlauf; die Gesetzesbegründungen sind insoweit unergiebig. ${ }^{77}$ Während die Vorschrift über die Klagebefugnis auf die Berührung des satzungsmäßigen Aufgabenkreises durch die Entscheidung abstellt (s. o.), ist im Rahmen der Begründetheit der Verstoß relevant, d. h. im Kontext des § 2 Abs. 5 UmwRG bzw. § 2 Abs. 4 UmwRG-E der Verstoß gegen entscheidungserhebliche Rechtsvorschriften. Dieser Verstoß muss die Belange berühren, deren Förderung der Verband sich verschrieben hat. Die Formulierung legt nahe - anders als im Rahmen der Zulässigkeit - nicht die Umweltauswirkungen des durch die Entscheidung zugelassenen Vorhabens in den Blick zu nehmen, sondern die Schutzrichtung der jeweils verletzten Rechtsvorschrift. Demnach wäre die Klage eines Vogelschutzverbands gegen die rechtswidrige Genehmigung einer Windenergieanlage, die Vögel tötet, unbegründet, wenn die Rechtswidrigkeit nicht auf einen Verstoß gegen vogelschutzrechtliche Vorschriften zurückzuführen ist. Von einer umfassenden Rechtmäßigkeitskontrolle könnte dann keine Rede sein: Nur qualifizierte Rechtsverstöße würden zur Aufhebung führen.

Sollte diese Lesart des $\S 2$ Abs. 4 Satz 1 a. E. UmwRG-E zutreffen - und der RegE legt dies nahe ${ }^{78}$-, würde die Frage nach der Vereinbarkeit mit Art. 9 Abs. 2 AK und der zu seiner Umsetzung ergangenen Richtlinien erneut aufgeworfen. Das ACCC hat sich hierzu im Verfahren gegen Deutschland nicht geäußert: Seine Ausführungen

\footnotetext{
${ }^{75}$ Vgl. Berkemann, DVB1. 2015, 389.

${ }_{76}$ Vgl. Ziekow, NVwZ 2007, 259, 262; Fellenberg/Schiller, in: Landmann/Rohmer, UmwR, § 2 UmwRG

Rdnr. 75 (Stand: April 2012).

${ }_{77}$ Vgl. BT-Drs. 16/2494, S. 13 r. Sp.; BT-Drs. 17/10957, S. 17 r. Sp.

${ }^{78}$ RegE, S. 36: „Im Ergebnis wird die Änderung damit nicht zur Rügefähigkeit jeglichen Rechtsverstoßes führen:"
} 
betreffen ausdrücklich nur die Regelung über die Klagebefugnis nach $\S 2$ Abs. 1 Nr. 2 UmwRG. Diese Vorschrift wird vom ACCC - zutreffend - als Kriterium über das „standing for judicial review“" verstanden, ${ }^{79}$ was so viel bedeutet wie „Klagebefugnis“. ${ }^{80}$ Das „standing“ des Umweltverbands darf davon abhängig gemacht werden, dass seine Ziele von der angegriffenen Entscheidung berührt werden („,are affected by the challenged decision“81). Auf diese Weise können die Vertragsstaaten den Kreis der Klageberechtigten begrenzen. ${ }^{82}$ Weitere Kriterien, die über die Regelung des „standings“ hinaus den Zugang zu gerichtlicher Kontrolle beschränken („that restrict access to the review procedure“), z. B. eine Begrenzung der Gründe, aus denen eine Entscheidung angefochten werden kann, sind hingegen konventionswidrig. ${ }^{83} \S 2$ Abs. 4 Satz 1 a. E. UmwRG-E lässt sich schwerlich als eine Regelung des „standings“ im Sinne einer Begrenzung des Kreises der Klageberechtigten ansehen. Denn er begrenzt den Prüfungsmaßstab, den das Gericht seiner Begründetheitsprüfung zugrunde legt, nachdem es die Zulässigkeit der Klage - und damit die Klagebefugnis/das „standing“ des Umweltverbands - bereits bejaht hat. Nimmt der Verband die Zulässigkeitshürde des § 2 Abs. 1 UmwRG, hat er „Zugang“ zum gerichtlichen Verfahren i. S. v. Art. 9 Abs. 2 AK erlangt. In der Folge geht es ausschließlich um die Prüfung der Rechtmäßigkeit der angefochtenen Entscheidung. Der Wortlaut der Konvention geht davon aus, dass der Kläger den Gerichtszugang erhält, um die Rechtmäßigkeit der angegriffenen Entscheidung voll infrage zu stellen (,to challenge the substantive and procedural legality of any decision"). Wie das ACCC und die Vertragsstaatenkonferenz im Verfahren gegen Deutschland festgestellt haben, dürfen die Vertragsstaaten den Umfang der dadurch eingeleiteten Prüfung nicht durch eine Qualifikation des Rechtsverstoßes beschränken, da es hierfür in Art. 9 Abs. 2 AK keine Grundlage gibt. Nichts anderes aber geschieht, wenn $\S 2$ Abs. 4 Satz 1 a. E. UmwRG-E die Begründetheit der Klage davon abhängig macht, dass der festgestellte Rechtsverstoß Belange berührt, für die der Umweltverband nach seiner Satzung eintritt. Diese Begrenzung der von Art. 9 Abs. 2 AK eingeforderten umfassenden Rechtmäßigkeitskontrolle - gleichsam "durch die Hintertür" - ist konventionswidrig und damit auch mit Art. 11 UVP-RL, Art. 25 IE-RL unvereinbar. Im Gesetzgebungsverfahren ist sie daher - jedenfalls für die Klagegegenstände nach $\S 1$ Abs. 1 Satz 1 Nr. 1 und 2 UmwRG - zu streichen.

\footnotetext{
${ }^{79}$ ACCC/C/2008/31, Tz. 71.

80 Vgl. Johnson, Nottingham LJ 2015, 137: „The test for standing is laid down in section 31(3) Senior Courts Act 1981 and requires the applicant to have ,sufficient interest' in the matter to which the application for permission to apply for judicial review relates."

${ }^{81} \mathrm{ACCC} / \mathrm{C} / 2008 / 31, \mathrm{Tz} .71$.

${ }^{82}$ ACCC/C/2008/31, Tz. 78.

83 ACCC/C/2008/31, Tz. 78.
} 


\subsection{Präklusion und Missbrauchsvorbehalt}

Mit der Aufhebung der materiellen Präklusionsregelungen des $\S 2$ Abs. 3 UmwRG, des $\S 10$ Abs. 3 Satz 5 BImSchG und der Präklusion nach § 47 Abs. 2a VwGO trägt der RegE den Vorgaben des EuGH Rechnung und geht sogar noch darüber hinaus, was Vorhaben und Pläne anbelangt, die nicht unter das einschlägige Richtlinienrecht fallen. Im Übrigen soll die eher kompliziert ausgestaltete Regelung die bestehenden Präklusionsvorschriften so weit wie möglich aufrechterhalten.

Dem anstelle der Präklusion eingeführten Missbrauchsvorbehalt in § 5 UmwRG-E dürfte in der Rechtspraxis allerdings nur geringe Bedeutung zukommen. Der Tatbestand - die erstmalige Geltendmachung ist „missbräuchlich oder unredlich“ ist denkbar unbestimmt. Die beiden Adjektive werden offenbar nicht wie Alternativen, sondern vielmehr gleich einem (missglückten) Hendiadyoin verwendet, um ein aus Gründen der Verfahrensökonomie missbilligtes Verhalten zu beschreiben. ${ }^{84}$ Für die Gerichte wird es schwierig sein, ein solches Verhalten im Einzelfall festzustellen. Die vom RegE angeführten Beispiele helfen nur bedingt weiter. Stets muss auch das Konventions- und Unionsrecht beachtet werden, das im Rahmen seines Anwendungsbereichs, einen weiten Zugang zu den Gerichten einfordert. Die Zurückweisung unredlichen oder missbräuchlichen Vorbringens ist, auch wenn der EuGH diese Möglichkeit offengehalten hat, eine Beschränkung des Gerichtszugangs. Wenn die Gerichte $§ 5$ UmwRG-E Konturen verleihen wollen, werden sie in letzter Konsequenz an Vorlagen zum EuGH nicht vorbeikommen, um sich ihre jeweilige Auslegung des § 5 UmwRG-E unionsrechtlich „absegnen“ zu lassen. Die Bereitschaft hierzu dürfte nicht allzu groß sein.

\section{Fazit}

Im Vergleich zu den bisherigen gesetzgeberischen „Bemühungen“ macht der RegE einen großen Schritt in Richtung konventions- und unionsrechtskonformer Zustände. Die Ausdehnung des Anwendungsbereichs der Umweltverbandsklage trägt den Vorgaben des Art. 9 Abs. 3 AK Rechnung. Die mit Art. 9 Abs. 2 AK unvereinbare Beschränkung des Kreises der rügefähigen Vorschriften im Rahmen der Klagebefugnis wird aufgegeben. Der Wegfall der Präklusionregelungen (nicht nur) im Anwendungsbereich der UVP- und IE-RL entspricht Art. 11 UVP-RL und Art. 25 IE-RL in der Auslegung des EuGH und geht sogar über deren Anwendungsbereich hinaus.

Bedenken sind freilich angebracht, soweit der RegE darauf abzielt, im Rahmen der Begründetheit den Kreis der rügefähigen Rechtsvorschriften - über eine Koppelung

${ }^{84}$ Vgl. RegE, S. 38 f. 
des Rechtsverstoßes an die Verbandssatzung - wieder einzuschränken. Dies ist mit Art. 9 Abs. 2 AK unvereinbar. Darüber hinaus bleibt abzuwarten, ob und mit welchen Änderungen der RegE nach Durchlaufen des parlamentarischen Verfahrens Gesetz wird. Die Entstehungs- und Änderungshistorie des UmwRG lässt insoweit „nichts Gutes“ erwarten. Da der Entwurf in dieser Form vom Kabinett verabschiedet worden ist, ist aber immerhin anzunehmen, dass das Unbehagen gegenüber der Umweltverbandsklage 10 Jahre nach Inkrafttreten des UmwRG der Einsicht gewichen ist, dass „halbherzige“ Lösungen dem völker- und unionsrechtlichen Anpassungsdruck nicht standhalten können. 